\title{
Exploring micromovements with motion capture and sonification
}

\author{
Alexander Refsum Jensenius ${ }^{\natural}$ and Kari Anne Vadstensvik Bjerkestrand \\ ${ }^{\natural}$ University of Oslo, Department of Musicology \\ a.r.jensenius@imv.uio.no, ka@bodymindflow.com
}

\begin{abstract}
We present the results of a pilot study on how micromovements may be used in an interactive dance/music performance. Micromovements are subtle body movements that cannot be easily seen by the human eye. Using an infrared marker-based motion capture system we have explored micromovements through 15x10 minutes long observation studies of ourselves standing still or moving slowly. The results from these studies show that there are both consistent and inconsistent movement patterns to be found at various temporal levels. Experimentation with three different types of realtime sonification shows artistic potential in using micromovements in dance and music performance.
\end{abstract}

Key words: micromovement, dance, motion capture, sonification

\section{Introduction}

Is it possible for humans to stand absolutely still? What types of micromovements are found when standing still? How can such micromovements be used in interactive dance/music performance? These are some of the questions we wanted to answer in the pilot project Sverm that we report on in this paper.

Our interest in, and methodological approaches to, the topic of micromovement are based on our backgrounds in music (first author) and dance (second author). Body movement is at the forefront in both music and dance, although for different reasons. The body movements of a musician are mainly soundproducing, while the movements of a dancer are often sound-accompanying. This traditional separation between musician and dancer is currently being challenged (see, e.g., [1]) with the increasing use of motion capture technologies that allow a dancer to create sound in realtime while moving, or for musicians to move more freely when playing.

The pilot project Sverm grew out of an interest in creating an interactive dance performance that would focus on the absence of movement: standstill. In our experience remarkably little is known about standstill in the fields of dance and music. Standstill is often regarded as uninteresting or the "fill" between actions or gestures, similar to how musicians and musicologists do not spend much time talking about silence. But in the same way that silence can be very powerful in music, as perhaps most famously documented in Cage's 4'33", we believe that standstill can be an interesting concept to work from in dance. 
But what is standstill? As living human beings, physical standstill is not possible to achieve. Most life processes, including in plants, in animals and in human beings, are carried out in recurring cycles [2. The periods of such cycles vary greatly: from the "nanomovement" of atoms in our cells, breathing and pulse cycles every few seconds, 24 hour sleep/wake cycles, yearly cycles in nature, and up to light-year cycles in the movement of planetary systems. Within this physical world, temporal coordination and adaptation are required for all living beings for survival, and this is the field of study in chronobiology. But how does the different chronobiological levels influence the performance and perception of music and dance?

We find it useful to differentiate between three different temporal levels: $m i$ cro, meso and macro, which coincide with the three main levels of our memory 3. The micro level describes events at the scale of milliseconds. Human experience within these ranges typically falls within the limits of the sensory memory, and is the basis for the experience of the other levels. The meso level falls within the boundaries of our short-term or working memory and constitutes most of human utterances (spoken words, musical phrases, etc.) and most everyday actions (opening a door, etc.). Finally, the macro level is founded in the long-term memory and is here used as a general term for all activities that are longer than the meso level. We should not forget that in the physical world there is a continuum from the smallest perceivable units to the largest. The experience of the micro, meso and macro levels is based on the limitations (and possibilities) of the human perceptual and cognitive system.

In dance and music the meso and macro levels are often the focus of attention: actions, gestures, phrases and large-scale form. The micro level, both spatially and temporally, is usually regarded more as fine-tuning of technique than as a meaning-bearing component in itself. However, there are some examples of composers and researchers that investigate microlevels in music, e.g., the exploration of microsound using granular sound synthesis techniques [4, and microrhythmic patterns and effects [5]. In the world of movement there are long traditions of slow movement in, e.g., Tai chi chuan, which inspired the second author's performance in the piece 10:50-11:00 [6]. But although such practice may also include micromovements to some extent, there are to our knowledge few examples of exploring the micromovements themselves, or standstill, in dance performance. Also interactive dance performances seem to focus mainly on large-scale movement, e.g., 7, 8. This has made us curious to see how micromovements, as observed in standstill, can be used in an interactive dance performance.

This paper starts by presenting a series of observation studies on standstill and slow movement that we have carried out to learn more about micromovements. This is followed by a presentation of some sonification strategies that may be used when working with such movements in creative practice. Finally, we suggest some possible directions in our future research and artistic activities. 


\section{Observation studies of micromovements}

To learn more about micromovements, we decided to carry out a series of observation studies where we would stand still or move slowly for 10 minutes at a time, as summarized in Table 1. First we did five standstill sessions, each with a slightly different mental strategy: attempting to stand physically still (1), mentally still (2), and two different types of imagined movement (3-4). All of these studies were done standing straight with the arms hanging down, but we also did one session with the arms straight in front of us (5). Next we did five recordings where we moved slowly in different patterns: shifting the weight back and forth between the legs (6), rotating the upper body (7), bending the knees (8), and head (only) movement in the three different planes (9-11). Finally, we did four more standstill studies (12-15) with the same mental strategies that we had used in the first recordings $(1-4)$.

Table 1. Overview of the 15 different 10-minute observation studies conducted

\begin{tabular}{|c|c|c|}
\hline \# Standstill & \#|Movement & \#|Standstill \\
\hline \begin{tabular}{c|l|}
1 & Physical standstill \\
\end{tabular} & $\begin{array}{l}6 \text { Weight shift (pendulum) } \\
\end{array}$ & \begin{tabular}{|c|c|}
12 & Physical standstill \\
\end{tabular} \\
\hline 2 Mental standstill & 7 Rotation (upper body) & 13 Mental standstill \\
\hline 3 Mental movement & 8 Knee bending & 14 Mental movement \\
\hline 4 Mental movement & 9 Head movement (sagittal) & 15 Mental movement \\
\hline 5 Arms in front & \begin{tabular}{|l|l}
10 & Head movement (pendulum) \\
11 & Head movement (rotation)
\end{tabular} & \\
\hline
\end{tabular}

\subsection{Method}

All sessions were carried out in the fourMs motion capture laboratories at the University of Oslo, and were recorded using an infrared marker-based motion capture system from Qualisys (9 Oqus 300 cameras) running at $100 \mathrm{~Hz}$. For the first sessions we only used a few markers each (on the neck, head and on the feet and hands), but added more markers in some of the later sessions. After each 10minute recording session we talked about the experience, and took notes of each other's immediate and subjective comments. These notes, together with analysis of the motion capture data carried out in Matlab using the MoCap toolbox [9], form the basis for our analysis of the material.

Before we started the study we thought that the standstill recordings would not be particularly interesting, and that they would mainly serve as a reference point for the recordings of movements. However, we have found the standstill recordings to be very interesting, so the following sections will mainly focus on some of these recordings (1-3 and 12-14). Furthermore, to constrain the analysis, we will mainly focus on the marker placed on the neck, at the cervical spinal \#7 (C7). The neck marker is particularly relevant, since it represents a point on the body that is fairly stable (more so than the head or hands), yet it reveals micromovements of the body very well. 


\subsection{How much movement?}

How still is it possible to stand? Our subjective experience was that we did move quite a lot: shifting weight between the legs, swaying, breathing, swallowing, etc. These movements were small, but they were easily noticeable to ourselves and to each other during the sessions.

Table 2.2 shows a summary of the distance travelled for two markers per person: one placed on the right foot (rFoot) and one placed on the neck $(\mathrm{C} 7)$. As a reference of the precision of the motion capture system, we have also included the distance travelled for a static marker placed on the floor next to us. Since this marker did not move at all, its value can be seen as the error margin of the recordings. This error is based on a combination of the resolution of the cameras and the calibration of the system. By comparing the movement of the feet to that of the reference marker, we see that the feet moved very little, and only marginally more than the reference. For recording 12, A-rFoot even moved less than the reference marker, but this is within the error margin of the system.

Table 2. Summary of the distance travelled (in meters) for markers on the right foot and at $\mathrm{C} 7$ for each of the 6 standstill studies.

\begin{tabular}{r|c|cc|cc} 
\# & Static & A-rFoot & A-C7 & KA-rFoot KA-C7 \\
\hline 1 & 1.10 & 1.15 & 2.85 & 1.19 & 5.89 \\
2 & 1.01 & 1.06 & 2.90 & 1.49 & 5.02 \\
3 & 1.09 & 1.25 & 2.76 & 1.41 & 5.39 \\
\hline 12 & 1.10 & 0.99 & 2.45 & 1.40 & 4.20 \\
13 & 0.95 & 0.99 & 2.72 & 1.17 & 3.79 \\
14 & 1.00 & 1.11 & 2.85 & 1.81 & 4.96
\end{tabular}

If we look at the results from the markers placed on the neck, A-C7 and KA-C7 respectively, we see that there is considerably more movement. Here we see that the results for the first author (A-C7) are generally quite low for all 6 recordings. The results for the three last recordings are slightly lower than for the first recordings, but not more than could be explained by the error margin. Even though the variation is larger for the second author (KA-C7), we see that she was standing more still in the three last recordings than in the first ones. This could be due to a heightened awareness developed over the course of the sessions, but it is difficult to draw any conclusions from this limited study.

By plotting the cumulative distances travelled for the above-mentioned markers, we see that the movement was more or less evenly distributed over the course of the 10-minute long sessions (Figure 1), as expected.

\subsection{Micro, meso and macro level activity}

Let us know turn to investigate the movement of a single marker for all sessions. Figure 2 shows plots of the neck marker (A-C7 and KA-C7) for recordings 1-3 and 12-14. The most important finding at the micro level is that there are, indeed, no signs of standstill: there is continuous movement at the scale of millimetres in all recordings. 


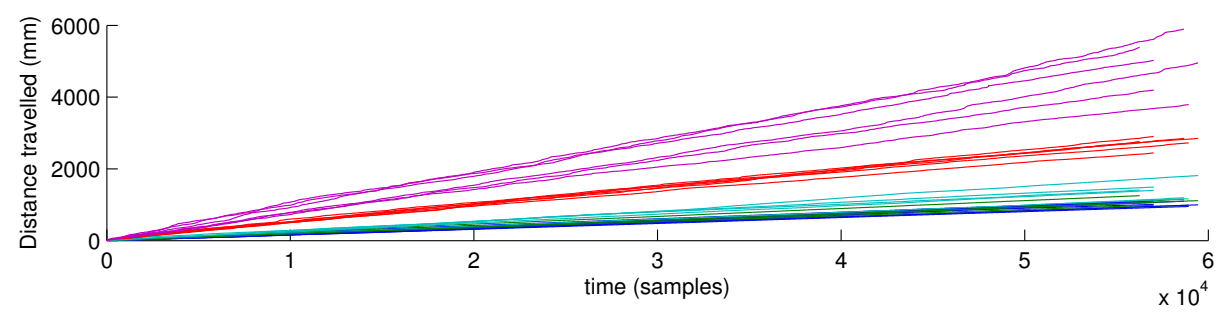

Fig. 1. Cumulative distances travelled for markers Static, A-rFoot, A-C7, KA-rFoot, $\mathrm{KA}-\mathrm{C} 7$ for recordings $1-3$ and $12-14$.
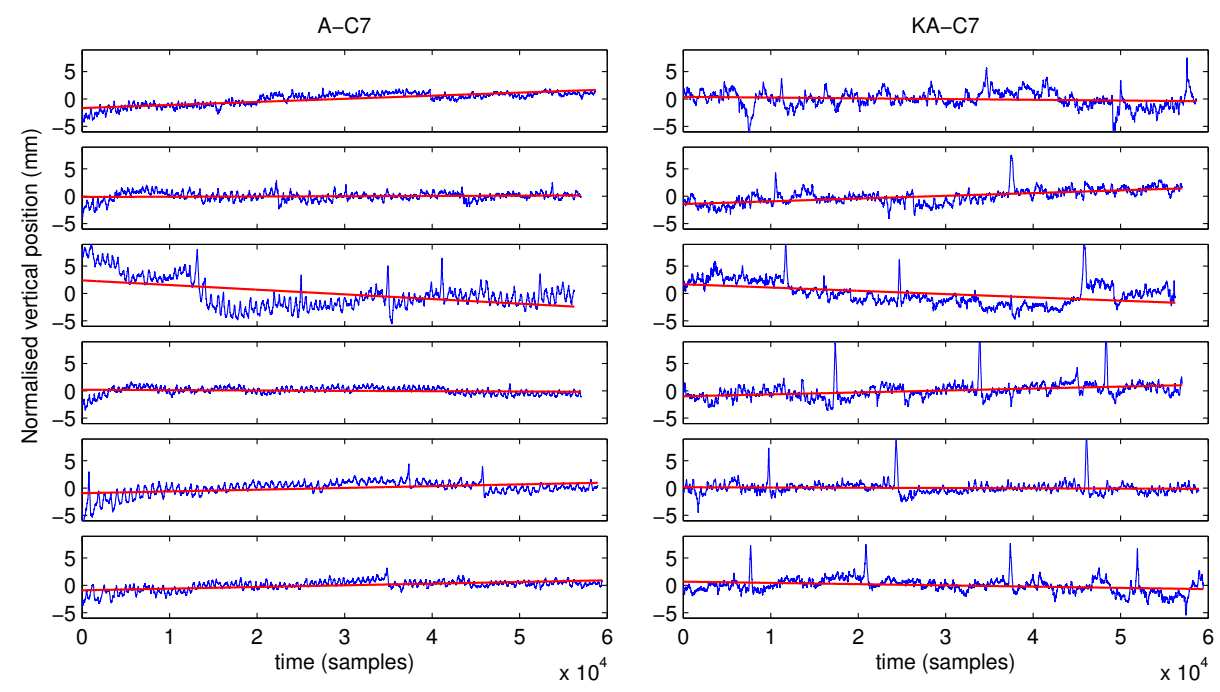

Fig. 2. Plots of the normalised vertical position of the neck marker $(\mathrm{C} 7)$ for recordings 1-3 and $12-14$ for the two authors (A and KA). The straight line indicates the slope of the data set.

At the meso level we can see from the plots that there are peaks in the signal approximately every $5-10$ seconds. Estimating the periodicity of the signals, using the mcperiod function in the MoCap Toolbox [10, reveals average periodicities in the vertical dimension at $6.0 \pm 1.4$ seconds and $9.1 \pm 2.5$ seconds for the two authors respectively. This periodicity most likely corresponds to the breathing pattern, and is something that it will be interesting to explore further in a performance setting.

At the macro level we see that linear regression gives a positive slope in 4 of 6 of the first author's sessions and 3 of 6 for the second author. We believe this can be explained by tension and/or fatigue, something we discussed several times in our post-recording talks. Sometimes we felt that we raised the shoulders because of fatigue, other times we worked towards lowering the shoulders as we straightened the back. This is also something that can be used as a feature in performance. 
The most surprising finding from the quantitative analysis is the "spikes" that can be seen in each recording. These are particularly prominent in the recordings of the second author, happening every $2-3$ minutes in all recordings. These spikes can probably be explained by postural readjustment, and we are very curious to see whether similar spikes can be seen also in other people standing still.

\subsection{Movement patterns}

What types of rotational movement patterns can be found? As the XY plots in Figure 3 show, more movement is happening along the Y-axis (front-back) than on the $\mathrm{X}$-axis (left-right). This can be explained by the feet stabilising the sideways movement more than the front-back movement.
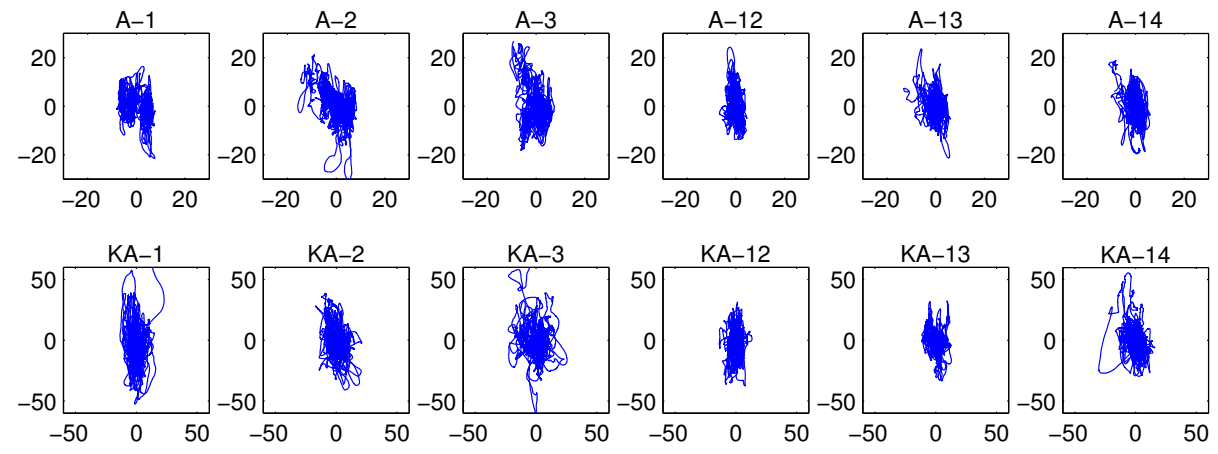

Fig. 3. XY movement patterns of the C7 marker from recordings 1-3 and 12-14 for the two authors (A and KA). The plots can be seen as the left-right (X-axis) and front-back (Y-axis, face pointing upwards) movement of the neck marker.

\section{Sonification of micromovements}

The subjective experience of the recording sessions, and subsequent analytical work, made us eager to start exploring how the micromovement data could be turned into sound through, i.e., sonification of the data. Since the aim of this work is to create an interactive dance performance, this exploration was mainly driven from an artistic point of view. Using the Qualisys motion capture system in realtime mode, we explored three different types of sonification:

- Sine tones controlled by the vertical position of individual markers.

- Noise controlled by the quantity of motion, with two different mappings: a) more motion produced more sound, b) less motion produced more sound.

- Sample playback using granular synthesis controlled by horizontal position. 
We started out by controlling a single sine tone with the vertical position of one marker. The aim was to see if we could "hear" the micromovements happening when standing still. For that reason we created a mapping from a small region $(5 \mathrm{~cm})$ to a large frequency range $(200-5000 \mathrm{~Hz})$. The effect was dramatic, as, e.g., breathing and heartbeats could easily be heard. We expanded the concept by adding more markers coupled to individual sine tones. This way it was possible to hear how different parts of the body moved at different paces (hands, shoulders, head). We also found it particularly interesting to place markers in close vicinity of each other, e.g., two markers on each hand, something that made it possible to play with interference patterns when the frequencies of the sine tones were close to each other.

For the second experiment, with white noise, we explored two strategies: either the level of noise would increase when there was more movement, or it would decrease. We found both of these approaches conceptually challenging to work with. Coupling sound level to quantity of motion is a logical mapping, as the sonification heightens the awareness of the movement. But we did not feel that this was particularly interesting to work with. Doing it the other way around, on the other hand, led to a very interesting interaction: the performer starts by moving in silence, and then as she approaches standstill the noise level increases. This created an interesting tension that we would like to explore further.

The third sonification experiment was based on controlling the playback point of short sound recordings by moving on the floor, similar to what has been explored in the Embodied Generative Music project [11]. Here the performer would move to a specific point on the floor, where she would spend several minutes working with micromovements to explore subtleties in the sound. The intuitive connection between location on the floor and the sound being played back gave the performer a sense of being in direct control of the sound, and was very inspiring to work with.

\section{Conclusions}

From the analysis of six of our standstill recordings we can conclude the following:

- It is not possible to stand still. Micromovements can be felt by the performer, can to some extent be seen by an observer, and can easily be picked up by a motion capture system.

- The level of standstill that it is possible to achieve is fairly stable and reproducible for one person.

- Different mental strategies for standing still did not change the quantitative results, but they changed the performer's own experience. Whether the mental strategy could be experienced by an observer should be investigated further.

- Breathing is the most important factor for movements at the temporal meso level, and postural adjustment at the temporal macro level. Periodicities at the temporal micro level need to be investigated further. 
The analytical findings will be used as the basis for creating movement-sound mappings in our interactive dance performance. Through our preliminary exploration of sonification strategies we can conclude:

- The standstill-noise mapping was the most interesting for exploring standstill, since the presence of sound heightened the sensation of standing still.

- It was interesting to explore beating patterns of sine tones when using two or more markers in close vicinity.

- The granulation experiment made it possible to explore microsound through micromovements very intuitively.

Future work include:

- conducting more standstill studies of ourselves, movement experts (musicians/dancers) and non-experts. The aim will be to discover more movement features that reliably can be used in performance.

- exploring how the different sonification strategies work with multiple performers, including testing out various types of sound spatialisation algorithms.

- exploring how our current ideas and technologies can be used in real performance in a theatre setting.

Acknowledgments. The project has been supported by Arts Council Norway, Norwegian Research Council, and University of Oslo.

\section{References}

1. Jensenius, A.R., Johnson, V.: Performing the electric violin in a sonic space. Computer Music Journal (In review)

2. Klein, G.: Farewell to the internal clock: a contribution in the field of chronobiology. Springer, New York, NY (2007)

3. Snyder, B.: Music and Memory: An Introduction. The MIT Press, Cambridge, MA (2000)

4. Roads, C.: Microsound. The MIT Press, Cambridge, MA (2001)

5. Danielsen, A., ed.: Musical Rhythm in the Age of Digital Reproduction. Ashgate, Farnham (2010)

6. Jacobsson, S., Bjerkestrand, K.A.V.: 10:50-11:00 [video] available from: http://youtu.be/y3gecc2_4xy (last checked: 10.11.2011) (2005)

7. Dobrian, C., Bevilacqua, F.: Gestural control of music: using the Vicon 8 motion capture system. In: Proceedings of the International Conference on New Interfaces for Musical Expression, Montreal (2003) 161-163

8. Downie, M.: Choreographing the Extended Agent: Performance Graphics for Dance Theater. PhD thesis, MIT, Cambridge, MA (2005)

9. Toiviainen, P., Burger, B.: MoCap Toolbox Manual. University of Jyväskylä, Jyväskylä, Finland (2010)

10. Eerola, T., Luck, G., Toiviainen, P.: An investigation of pre-schoolers' corporeal synchronization with music. In: Proceedings of the 9th International Conference on Music Perception \& Cognition, Bologna. (2006) 472-476

11. Peters, D.: Enactment in listening: Intermedial dance in EGM sonic scenarios and the bodily grounding of the listening experience. Performance Research 15 (2010) $81-87$ 